\title{
"Grandiosity" as a signature symptom for Bipolarity in Non-Bipolar Depression. WCP19-1519
}

G. Anmella ${ }^{1}$, J. Gil-Badenes ${ }^{1}$, I. Pacchiarotti ${ }^{1}$, N. Verdolini ${ }^{1,2,3}$, A. Aedo ${ }^{1,4}$, F. Corponi ${ }^{1}$, M. Muscas ${ }^{1}$, J. Pinzon ${ }^{1}$, A. Gimenez¹, S. Gomes ${ }^{1}$, C. Llach¹, E. Vieta ${ }^{1}$, A. Murru for the BRIDGE-II-Mix Study Group.

'Bipolar Disorder Unit, Institute of Neuroscience, Hospital Clinic, University of Barcelona, IDIBAPS, CIBERSAM, Barcelona, Catalonia, Spain.

2Santa Maria della Misericordia Hospital- University of Perugia, Division of Psychiatry- Clinical Psychology and Rehabilitation- Department of Medicine, Perugia, Italy.

${ }^{3}$ FIDMAG, Germanes Hospitalàries Research Foundation, Sant Boi de Llobregat- Barcelona- Catalonia- Spain, Spain.

${ }^{4}$ School of Medicine. Pontificia Universidad Católica de Chile, Bipolar Disorders Unit- Department of Psychiatry, Santiago, Chile.

\section{Introduction}

Depressive mixed state is present in around $24 \%$ of unipolar depressed (UD) patients [1,2]. "Grandiosity", although being a rare symptom in mixed depression, is included as a core (hypo)manic diagnostic feature in the DSM-5 "mixed features" specifier [3]. Some studies defined grandiosity as a maladaptive cognition associated with bipolarity $[4,5]$ even outlining grandiosity as an early-diagnostic bipolar feature [6].

\section{Aim}

To evaluate the likelihood of "grandiosity" in predicting worse functioning and its association with bipolar diathesis in UD and first depressive episode (FDE) patients.

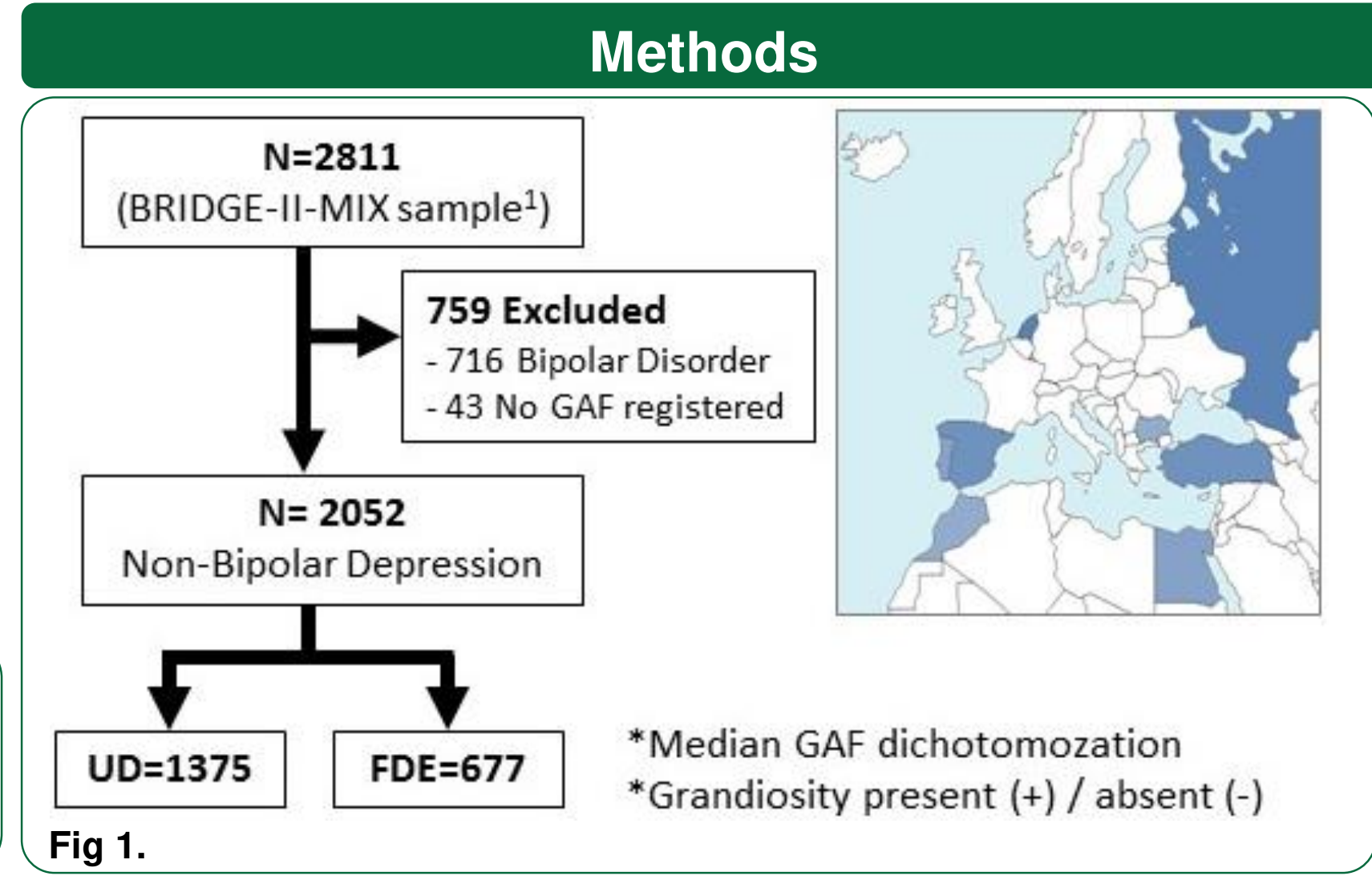

A post-hoc analysis of the BRIDGE-II-Mix study. A total of 2052 acutely depressed patients (UD=1375 and FDE=677) were stratified in grandiosity+ and grandiosity- (Fig 1). Functioning was evaluated using the median GAF score as a dichotomizing cutoff. Statistical analyses considered Bonferroni's adjustment for multiple comparisons $(n=20)$, set to $p=0.0025$.

\section{Results}

"Grandiosity" prevalence was $<\mathbf{2 . 5 \%}$ both in UD and FDE. Stratification: Grandiosity+ (n=37, $1.80 \%$ )/Grandiosity- (n=2051, 98.20\%) (Fig 2).

\section{Non-Bipolar Depression}

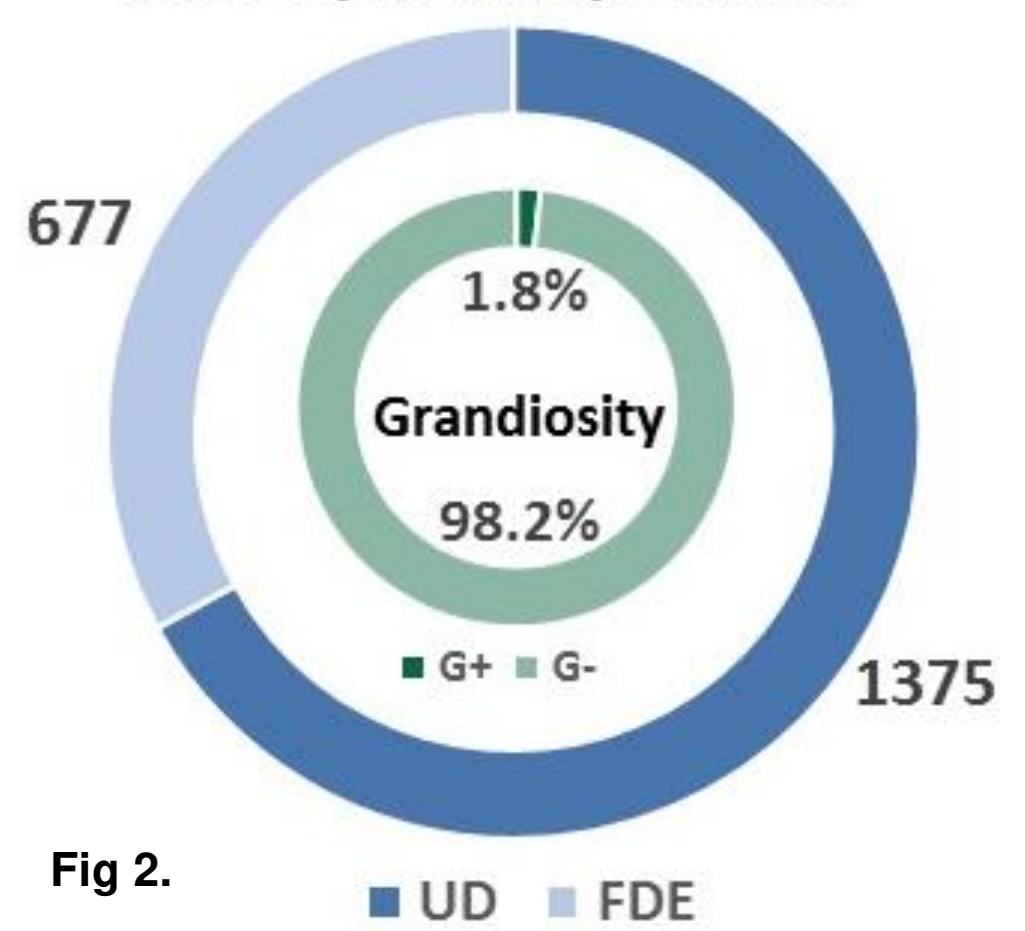

Grandiosity+ patients were younger, presented with earlier disease onset, worse clinical evolution and functioning and more mixed symptoms (see Tab 1).
Tab. 1. Differences among Grandiosity +/-

\begin{tabular}{c|c|c}
\hline Grandiosity+ & Grandiosity- & p \\
$\mathrm{n}=37,1.80 \%$ & $\mathrm{n}=2051,98.20 \%$
\end{tabular}

\begin{tabular}{|c|c|c|c|}
\hline Age & & & \\
\hline Current Age, mean $\pm S D$ & $37.97 \pm 12.92$ & $44.04 \pm 14.01$ & 0.009 \\
\hline Age $1^{\text {st }}$ symptoms, mean $\pm S D$ & $26.30 \pm 12.21$ & $34.36 \pm 13.15$ & $<0.0001$ \\
\hline Severity of depression & & & \\
\hline $\mathrm{N}$ past depressive episodes & $8.08 \pm 14.47$ & $3.67 \pm 4.20$ & $<0.0001$ \\
\hline Tot suicidal attempts & $1.27 \pm 3.46$ & $0.33 \pm 0.88$ & $<0.0001$ \\
\hline Mixicity & & & \\
\hline Total (hypo)manic sympt & $7.59 \pm 2.91$ & $1.33 \pm 2.04$ & $<0.0001$ \\
\hline Functioning & & & \\
\hline GAF scores, mean $\pm S D$ & $45,68 \pm 12.97$ & $51,90 \pm 12.43$ & 0.003 \\
\hline Worse functioning ${ }^{1}$ & $n=28,75.7 \%$ & $n=852,42.3 \%$ & $<0.0001$ \\
\hline
\end{tabular}

Conclusions

1. Non-bipolar depressed (UD and FDE) patients with "grandiosity" represent a differential significant more severe subgroup in terms of sociodemographic, clinical factors and a markedly worse functioning comparing to the non-grandiosity subgroup.

2. This subgroup shares common characteristics with bipolar patients, thus pointing to a bipolar diathesis and highlighting "grandiosity" as a possible predictor of bipolarity in UD and FDE.

\section{References}

Perugi G, et al. Mixed Features in Patients With a Major Depressive Episode. J Clin Psychiatry. 2015

Vázquez GH, et al. Mixed symptoms in major depressive and bipolar disorders: A systematic review. J Affect Disord. 2018

Solé E, et al. Mixed features in bipolar disorder. CNS Spectr. 2017

Goldberg JF, et al. Content-specificity of dysfunctional cognitions for patients with bipolar mania versus unipolar depression: a preliminary study. Bipolar Disord. 2005

Hawke LD, et al. Early Maladaptive Schemas among patients diagnosed with bipolar disorder. J Affect Disord. 2012

6. Ferreira-Maia AP, et al. Evaluation of Bipolar Disorder in Children and Adolescents Referred to a Mood Service. J Psychiatr Pract. 2016.

\section{Confilicts of Interest}

Dr. Vieta reports grants and/or fees from $A B$ Biotics, Abbott, Allergan, Angelini, Dainippon Sumitomo Pharma, Ferrer, Geodon Richter Janssen, Lundbeck, Otsuka, SAGE, Sanofi, Sunovion, and Takeda.

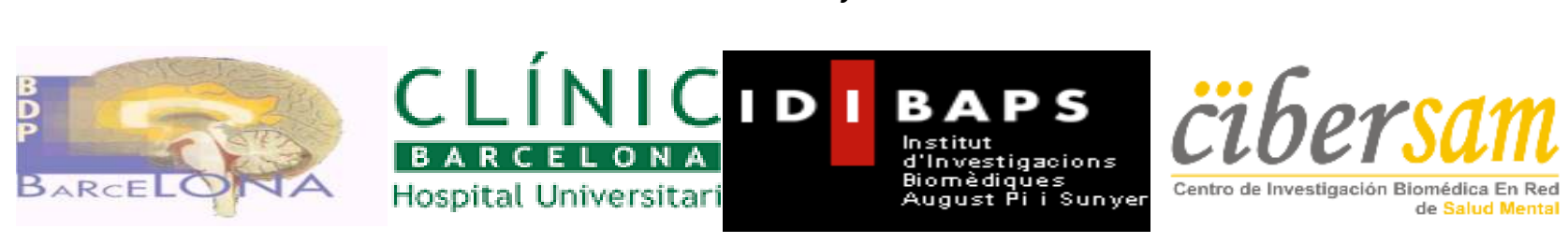

